\title{
A LOWER BOUND FOR SECTIONAL GENERA OF AMPLE AND SPANNED VECTOR BUNDLES ON ALGEBRAIC SURFACES
}

\author{
ANTONIO LANTERI AND FRANCESCO RUSSO
}

(Communicated by Eric Friedlander)

\begin{abstract}
Let $E$ be an ample and spanned vector bundle of rank $r$ over a complex projective surface $S$. It is shown that the sectional genus $g(S, \operatorname{det} E)$ is bounded from below by the number $b=\max \left\{q(S), 2-r \chi\left(\mathscr{O}_{S}\right)\right\}$ and pairs $(S, E)$ satisfying $b \leq g(S, \operatorname{det} E) \leq b+1$ are characterized.
\end{abstract}

\section{INTRODUCTION}

Let $E$ be an (algebraic) ample vector bundle of rank $r$ on a complex projective smooth surface $S$ and let $\operatorname{det} E=\Lambda^{r} E$ (ampleness has to be intended as in [Ha1]). The sectional genus of $E$ is defined by the adjunction formula

$$
2 g(S, \operatorname{det} E)-2=c_{1}(E) \cdot\left(c_{1}(E)+K_{S}\right),
$$

where $K_{S}$ is the canonical bundle of $S$, and satisfies $g(S, \operatorname{det} E) \geq 0$. Recent papers [F1, BLL] focused the interest on the classification of ample vector bundles with low sectional genera. Assume that $E$ is ample and spanned, i.e., the locally free sheaf $\mathscr{O}_{S}(E)$ is generated at every point by global sections; then $\operatorname{det} E$ is an ample and spanned line bundle, hence the linear system $|\operatorname{det} E|$ contains a smooth curve, whose geometric genus is $g(S, \operatorname{det} E)$. Thus we have the obvious inequality $g(S$, det $E) \geq q(S) \quad\left(=h^{1}\left(\mathscr{O}_{S}\right)\right)$ resulting, e.g., from the Lefschetz theorem (see [So]). This inequality can be considerably improved, at least when $S$ is a ruled surface. In fact the aim of this paper is to prove the following

(0.1) Theorem. Let $E$ be an ample and spanned rank-r vector bundle $(r \geq 2)$ on a complex projective smooth surface $S$. Then

(a) $g(S, \operatorname{det} E) \geq \max \left\{q(S), 2-r \chi\left(\mathscr{O}_{S}\right)\right\}$ and if equality holds, then $(S, E) \simeq$ $\left(\mathbf{P}^{2}, \mathcal{O}_{\mathbf{P}}(1)^{\oplus 2}\right)$.

Moreover,

(b) If $g(S, \operatorname{det} E)=\max \left\{q(S)+1,3-r \chi\left(\mathscr{O}_{S}\right)\right\}$ then $(S, E)$ is one of the following pairs:

(b1) $\left(\mathbf{P}^{2}, \mathscr{O}_{\mathbf{P}^{2}}(1) \oplus \mathscr{O}_{\mathbf{P}^{2}}(2)\right)$,

Received by the editors April 2, 1992.

1991 Mathematics Subject Classification. Primary 14J60; Secondary 14C20.

Key words and phrases. Surface (complex projective), vector bundle (ample), sectional genus, adjunction. 
(b2) $\left(\mathbf{P}^{2}, \mathscr{O}_{\mathbf{P}^{2}}(1)^{\oplus 3}\right)$,

(b3) $\left(\mathbf{P}^{2}\right.$, tangent bundle),

(b4) $\left(\mathbf{P}^{1} \times \mathbf{P}^{1}, \mathscr{O}_{\mathbf{P}^{1} \times \mathbf{P}^{1}}(1,1)^{\oplus 2}\right)$,

(b5) $S=\mathbf{P}(F)$ is a $\mathbf{P}^{1}$-bundle over an elliptic curve $B$ and $E=H(F) \otimes \pi^{*} G$, where $\pi: S \rightarrow B$ is the ruling projection, $F$ and $G$ are indecomposable vector bundles of rank 2 on $B$ with $c_{1}(F)=c_{1}(G)=1$, and $H(F)$ denotes the tautological bundle of $F$.

To point out a corollary on the sectional genus of some special polarized manifolds, let us recall the following definition. A pair $(P, H)$ consisting of a complex projective manifold $P$ and an ample line bundle $H \in \operatorname{Pic}(P)$ is said to be a scroll (in the classical sense) over $S$ if $P$ is a $\mathbf{P}^{r-1}$-bundle over $S$ and $H$ induces $\mathscr{O}_{\mathbf{P}^{r-1}}(1)$. on every fiber. Let $p: P \rightarrow S$ be the bundle projection and set $E=p_{*} H$. Then $E$ is a rank- $r$ vector bundle on $S$, whose tautological line bundle $H(E)$ on $P$ is exactly $H$. Therefore $E$ is ample since $H$ is ample. Moreover, since $S$ is a surface, we have $g(P, H)=g(S, \operatorname{det} E)$ [F1, (1.2)]. Thus $(0.1)(\mathrm{a})$ gives

$(0.2)$ Corollary. Let $(P, H)$ be a scroll over a smooth surface $S$, the polarizing line bundle $H$ being ample and spanned. Then

$$
g(P, H) \geq \max \left\{q(S), 2-r \chi\left(\mathscr{O}_{S}\right)\right\},
$$

equality implying that $(P, H) \simeq\left(\mathbf{P}^{2} \times \mathbf{P}^{1}, \mathscr{O}_{\mathbf{P}^{2} \times \mathbf{P}^{1}}(1,1)\right)$.

Similar characterizations can be deduced from $(0.1)(b)$.

\section{Preliminary lemmas}

We use the standard notation in algebraic geometry. Following current abuses we do not distinguish between line bundles and invertible sheaves and use the additive notation for the tensor product of line bundles. The symbol $\equiv$ will stand for numerical equivalence. We denote by $c_{t}(E)$ the Chern polynomial of $E$. This section is devoted to some lemmas we need to prove $(0.1)$.

(1.1) Lemma. Let $E$ be an ample vector bundle of rank $r$ on $S$ and let $C$ be a smooth rational curve on $S$. Then $\operatorname{deg} E_{C} \geq r$, equality implying $E_{C} \simeq$ $\mathscr{O}_{\mathbf{P}^{1}}(1)^{\oplus r}$.

Proof. Since $C \simeq \mathbf{P}^{1}$ we have $E_{C}=\bigoplus_{i=1}^{r} \mathscr{O}_{\mathbf{P}^{1}}\left(a_{i}\right)$, where $a_{i}>0$ due to the ampleness. Hence $\operatorname{deg} E_{C}=\sum a_{i} \geq r$ and equality implies $a_{i}=1$ for each $i$.

(1.2) Lemma [LM, Lemma 4]. Let $E$ be an ample and spanned vector bundle of rank $r$ on $S$ with $c_{2}(E)=1$. Then $(S, E) \simeq\left(\mathbf{P}^{2}, \mathscr{O}_{\mathbf{P}^{2}}(1)^{\oplus 2}\right)$.

(1.3) Lemma. Let $S$ be a $\mathbf{P}^{1}$-bundle over a smooth curve of genus $q$ and let $E$ be a vector bundle of rank $r \geq 2$ on $S$ such that $E_{f} \simeq \mathscr{O}_{\mathbf{P}^{1}}(1)^{\oplus r}$ for every fiber $f$ of $S$. Then $g(S, \operatorname{det} E)=r(q-1)+c_{2}(E)+1$.

Proof. Let $S=\mathbf{P}_{B}(F)$ and let $h=H(F)$ denote the tautological line bundle of the rank-2 vector bundle $F$ on the base curve $B$. We also assume that $F$ is normalized as in [Ha2, Chapter 5]. Let $e=-h^{2}$ be the invariant of $S$. Since $\left(E \otimes[h]^{-1}\right)_{f} \simeq \mathscr{O}_{\mathbf{P}^{1}}^{\oplus r}$, we have that $E \otimes[h]^{-1}=\pi^{*} G$, where $\pi$ is the ruling projection and $G$ is a rank-r vector bundle on $B$. Hence

$$
E=[h] \otimes \pi^{*} G \text {. }
$$


Computing the Chern classes of $E$ we get

$$
c_{2}(E)=(r-1)\left(\gamma-\frac{r e}{2}\right),
$$

where $\gamma=\operatorname{deg} G$. Actually, letting $c_{t}\left(\pi^{*} G\right)=\left(1+\gamma_{1} t\right) \cdots\left(1+\gamma_{r} t\right)$ with $\gamma_{1}+$ $\cdots+\gamma_{r}=c_{1}\left(\pi^{*} G\right) \equiv \gamma f$, and recalling that $c_{t}(E)=\prod_{i<j}\left(1+\left(h+\gamma_{i}\right) t\right)$, we obtain

$$
\begin{aligned}
c_{2}(E) & =\sum_{i<j}\left(h+\gamma_{i}\right) \cdot\left(h+\gamma_{j}\right)=\sum_{i<j}\left(h^{2}+h \cdot\left(\gamma_{i}+\gamma_{j}\right)+\gamma_{i} \cdot \gamma_{j}\right) \\
& =\frac{r(r-1)}{2} h^{2}+(r-1) \gamma h \cdot f=(r-1)\left(\gamma-\frac{r e}{2}\right) .
\end{aligned}
$$

Let $L=\operatorname{det} E$; then $L \equiv r h+\gamma f$. On the other hand, $K_{S} \equiv-2 h+(2 q-2-e) f$. So we get

$$
L \cdot\left(L+K_{S}\right)=(r-1)(2 \gamma-e r)+2 r(q-1),
$$

and then recalling (1.3.1) by the genus formula we obtain

$$
2 g(S, \operatorname{det} E)-2=2 r(q-1)+2 c_{2}(E),
$$

which proves the assertion.

(1.4) Lemma. Let $E$ be an ample and spanned rank-r vector bundle on a surface $S$. Equality $g(S, \operatorname{det} E)=3-r \chi\left(\mathscr{O}_{S}\right)$ cannot occur if $S$ is a $\mathbf{P}^{1}$-bundle over a smooth curve of genus $q \geq 2$.

Proof. Let $S, h, f$, and $e$ be as above and by contradiction assume that $g(S, \operatorname{det} E)=3+r(q-1) ; \operatorname{set} L=\operatorname{det} E$ and $m=L \cdot f$. Since $L \equiv m h+b f$ and $K_{S} \equiv-2 h+(2 q-2-e) f$ by the genus formula we have

$$
4=(m-1)(2 b-m e)+2(m-r)(q-1) .
$$

Of course $m \geq r$ by (1.1). If $m=r$, then $S$ is a $\mathbf{P}^{1}$-bundle and by (1.3) we get $g(S, \operatorname{det} E)=r(q-1)+c_{2}(E)+1$, so that $c_{2}(E)=2$. Using the Serre exact sequence for spanned bundles as in [OSS, p. 81]

$$
0 \rightarrow \mathscr{O}_{S}^{\oplus(r-2)} \rightarrow E \rightarrow F \rightarrow 0,
$$

we see that $2=c_{2}(E)=c_{2}(F)$, where $F$ is an ample and spanned rank-2 vector bundle on $S$. But this gives a contradiction, since given that $q \geq 2$, it must be that $c_{2}(F) \geq 3$ by [N, Theorem 6.1 ; BaL]. We can thus assume $m \geq r+1 \geq 3$. Note that $2 b-m e>0$ by the ampleness assumption [Ha2, Chapter 5]. So, as $q \geq 2$, we have

$$
2(2 b-m e)+2(q-1) \geq 4,
$$

which combined with (1.4.1) implies $m=r+1=3$ with $q=2$; in this case we get $2 b-m e=1$, i.e., $b=\frac{m e+1}{2}=\frac{3 e+1}{2}$ and from the ampleness condition for $L$ we get $e=-1$. On the other hand, since $E$ is an ample and spanned rank-2 vector bundle and $h$ is a smooth curve of genus $q=2,[\mathrm{BaL},(1.1)]$ implies $\operatorname{deg} E_{h} \geq 4$. So we have

$$
4 \leq \operatorname{deg} E_{h}=L \cdot h=(3 h-f) \cdot h=3-1=2,
$$

a contradiction. 


\section{PROOF OF THE THEOREM}

(2.1) Proof of (a). We let $g=g(S$, det $E)$ for brevity. We have $g \geq q(S)$ as observed in the introduction. Suppose equality holds. By the Riemann-Roch and the Kodaira vanishing theorems we get $h^{0}\left(K_{S}+\operatorname{det} E\right)=g-q(S)+p_{g}(S)=$ 0 ; indeed if $p_{g}(S)>0$, from $\operatorname{dim}\left|K_{S}+\operatorname{det} E\right| \geq \operatorname{dim}\left|K_{S}\right|+\operatorname{dim}|\operatorname{det} E|$ and from the fact that $h^{0}(\operatorname{det} E) \geq 3$, due to the spannedness of $\operatorname{det} E$, we would obtain

$$
g \geq q(S)+h^{0}(\operatorname{det} E)-1>q(S)+1,
$$

a contradiction. Hence $K_{S}+\operatorname{det} E$ is not spanned and then $(S, E) \simeq$ $\left(\mathbf{P}^{2}, \mathscr{O}_{\mathbf{P}^{2}}(1)^{\oplus 2}\right)$ by [LM, Theorem A; R, (3.1)]. Assume $\chi\left(\mathscr{O}_{S}\right)>0$. Then since $r \geq 2$ we have $2-r \chi\left(\mathscr{O}_{S}\right) \leq 0 \leq q(S)$ and so there is nothing to prove. If $\chi\left(\mathscr{O}_{S}\right)=0$, we always have $g \geq 2$ as follows from [BLL, (1.1)-(1.3)]. So we can suppose $\chi\left(\mathscr{O}_{S}\right)<0$ and then $S$ is a ruled surface with $q=q(S) \geq 2$ by the Castelnuovo-De Franchis theorem. Let $\pi: S \rightarrow B$ be the ruling projection of $S$ over the smooth base curve $B$ of genus $q$. Since det $E$ is ample and spanned there exists a smooth curve $C$ in the linear system $|\operatorname{det} E|$, whose geometric genus is $g$. Let $\alpha: C \rightarrow B$ be the restriction of $\pi$ to $C$. Since $C$ is ample, $\alpha$ is surjective; let $m$ be its degree. If $f$ is a general fiber of $\pi$ we have

$$
m=C \cdot f=c_{1}\left(E_{f}\right) ;
$$

hence $m \geq r$ by Lemma (1.1) and equality implies both that $S$ is a $\mathbf{P}^{1}$-bundle and that $E_{f} \simeq \mathscr{O}_{\mathbf{P}^{1}}(1)^{\oplus r}$ for every fiber $f$. So if $m=r$ by Lemmas (1.2) and (1.3) we get

$$
g \geq r(q-1)+3=3-r \chi\left(\mathscr{O}_{S}\right) .
$$

So we can assume $m \geq r+1$. By the Riemann-Hurwitz formula applied to $\alpha$ we thus obtain

$$
\begin{aligned}
g & \geq m(q-1)+1 \geq(r+1)(q-1)+1 \\
& \geq r(q-1)+q \geq r(q-1)+2=2-r \chi\left(\mathscr{O}_{S}\right),
\end{aligned}
$$

which gives the desired inequality. Moreover if $g=2-r \chi\left(\mathscr{O}_{S}\right)$, then $q=2$ and $m=r+1$. Note that in this case $S$ is a $\mathbf{P}^{1}$-bundle as well. Actually, if $f_{1}, f_{2}$ were two irreducible components of a reducible fiber, we would have

$$
r+1=m \geq c_{1}\left(E_{f_{1}}\right)+c_{1}\left(E_{f_{2}}\right) \geq 2 r,
$$

contradicting $r \geq 2$. Therefore $S=\mathbf{P}_{B}(F)$ with $F$ a rank-2 vector bundle over $B$. Let $h=H(F)$ and suppose that $F$ is normalized. We have $\operatorname{det} E \equiv$ $(r+1) h+b f$ for some integer $b$. Since $K_{S} \equiv-2 h+(2-e) f$, we get by the genus formula

$$
2 r+2=2 g-2=r(2 b-(r+1) e)+2 r+2,
$$

i.e.,

$$
r(2 b-(r+1) e)=0
$$

contradicting the ampleness of $\operatorname{det} E$.

(2.2) Proof of (b). If $g=q(S)+1$, we can suppose $p_{g}(S)=0$ by $(2.1 .1)$; then $h^{0}\left(K_{S}+\operatorname{det} E\right)=1$ and $K_{S}+\operatorname{det} E$ is spanned by [LM, Theorem A]; so $S$ is a Del Pezzo surface and $g=1$. Then the pairs in (b1)-(b4) follow from [F1, 
(1.5); R, (3.2)]. If $\chi\left(\mathscr{O}_{S}\right) \geq 0$, use [BLL] and get (b5). If $\chi\left(\mathscr{O}_{S}\right)<0$, then $S$ is a ruled surface with $q \geq 2$. As in the proof of Lemma (1.4) we can assume $c_{2}(E) \geq 3$. Let $\pi, \alpha$, and $m$ be as above. As before $m \geq r$ and the case $m=r$ cannot hold by Lemma (1.3). So we can assume $m \geq r+1$ and by the Riemann-Hurwitz formula again we see that $S$ must be a $\mathbf{P}^{1}$-bundle except in the following case:

- $S$ is a ruled surface over a smooth curve of genus $q=2$, having some reducible fiber consisting of two irreducible components, $r=2, m=4$, $g(S, \operatorname{det} E)=5, c_{2}(E) \geq 3$.

Since the $\mathbf{P}^{1}$-bundle case is settled by Lemma (1.4), it only remains to exclude the above case. To do this, note that the line bundle $K_{S}+L$, where $L=\operatorname{det} E$, is ample by [F2] and spanned by [LM, Theorem A]. By the Riemann-Roch and the Kodaira vanishing theorems we get $h^{0}\left(K_{S}+L\right)=g-q=3$, hence the map associated to $\left|K_{S}+L\right|$ is a finite morphism $\phi: S \rightarrow \mathbf{P}^{2}$. Since $S$ is irregular, $\phi$ cannot have degree $\leq 2$ and so $\left(K_{S}+L\right)^{2}=\operatorname{deg} \phi \geq 3$. Moreover, since $S$ is obtained from a minimal model by blowing up $\nu \geq 1$ points, we have $K_{S}^{2}=8(1-q)-\nu<-8$. Using the genus formula we thus get

$$
3 \leq\left(K_{S}+L\right)^{2}=K_{S}^{2}+4(g-1)-L^{2}<8-L^{2},
$$

i.e., $c_{1}(E)^{2}<5$. Now consider the pair $(P=\mathbf{P}(E), H=H(E))$. Since $d(P, H)=c_{1}(E)^{2}-c_{2}(E)$ (e.g., see $[\mathrm{F} 1,(2.2)]$ ) we get $d(P, H)<3$. Since $H$ is ample and spanned, if $d(P, H)=1$ then $P$ is isomorphic to $\mathbf{P}^{3}$, while if $d(P, H)=2$, then $P$ is either isomorphic to a quadric threefold, or a double cover of $\mathbf{P}^{3}$; but in all these cases $P$ would be simply connected, contradicting the fact that $b_{1}(P)=b_{1}(S)=2 q=4$.

\section{FURTHER REMARKS AND COMMENTS}

Looking over the proof of Theorem $(0.1)(a)$ we see that the spannedness assumption is used three times: a first time for the need of Lemma (1.2), a second time to have $h^{0}(\operatorname{det} E) \geq 3$, and a third time for the Riemann-Hurwitz argument. Actually we do not always need the spannedness of $E$ but simply the facts that $|\operatorname{det} E|$ is at least a pencil in the second case and that $|\operatorname{det} E|$ contains a smooth curve in the third one. In fact the last assumption is exactly what we need in the proof of the inequality in Theorem $(0.1)(a)$. We do not know whether the lower bound $\max \left\{q(S), 2-r \chi\left(\mathscr{O}_{S}\right)\right\}$ continues to hold if $E$ is simply assumed to be an ample vector bundle. However the spannedness assumption is crucial for Lemma (1.2), as the following example shows.

(3.1) Example. Let $S=\mathbf{P}_{B}(F)$, where $F$ is an indecomposable rank-2 vector bundle on a smooth curve $B$ of genus $q \geq 1$ with $\operatorname{deg} F=1$ and let $h=$ $H(F)$. The rank-2 vector bundle $E=[h]^{\oplus 2}$ is ample but not spanned, although $\operatorname{det} E=[2 h]$ is spanned for $q=1$. We have $c_{2}(E)=h^{2}=1$. Note also that $g(S, \operatorname{det} E)=2 q=2-r \chi\left(\mathscr{O}_{S}\right)$.

For more examples of ample but not spanned vector bundles $E$ with $c_{2}(E)=$ 1 see $[\mathrm{F} 1,(2.18)$ and $(2.4)$, subcase $f=0]$.

The inequality in $(0.1)$ was suggested by the following inequality holding for decomposable vector bundles. 
(3.2) Proposition. Let $E=\bigoplus_{i=1}^{r} L_{i}$ be a decomposable ample and spanned vector bundle on a surface $S$. Then

$$
g(S, \operatorname{det} E) \geq r(q(S)-1)+c_{2}(E)+1
$$

and equality implies that either $(S, E) \simeq\left(\mathbf{P}^{2}, \mathscr{O}_{\mathbf{P}^{2}}(1)^{\oplus p} \oplus \mathscr{O}_{\mathbf{P}^{2}}(2)^{\oplus(r-p)}\right) \quad(0 \leq$ $p \leq r)$ or $S$ is a $\mathbf{P}^{1}$-bundle over a smooth curve and $E_{f} \simeq \mathscr{O}_{\mathbf{P}^{1}}(1)^{\oplus r}$ for every fiber $f$ of $S$.

Proof. From

$$
c_{1}(\operatorname{det} E)=c_{1}(E)=\sum_{i=1}^{r} c_{1}\left(L_{i}\right), \quad c_{2}(E)=\sum_{i<k} c_{1}\left(L_{i}\right) \cdot c_{1}\left(L_{k}\right),
$$

and the genus formula we get

$$
g(S, \operatorname{det} E)=g\left(S, L_{1}\right)+\cdots+g\left(S, L_{r}\right)+c_{2}(E)-(r-1) ;
$$

since $L_{i}$ is ample and spanned we have $g\left(S, L_{i}\right) \geq q(S)$ and so

$$
g(S, \operatorname{det} E) \geq r q(S)+c_{2}(E)-r+1,
$$

equality implying $g\left(S, L_{i}\right)=q(S)$ for every $i$. By [LP, (3.2)] we see that either $\left(S, L_{i}\right) \simeq\left(\mathbf{P}^{2}, \mathscr{O}_{\mathbf{P}^{2}}(a)\right)$ with $a=1,2$ or $S$ is a $\mathbf{P}^{1}$-bundle and $L_{i f} \simeq \mathscr{O}_{\mathbf{P}^{1}}(1)$ for every fiber $f$ of $S$ and so we are done.

(3.3) Remarks. (i) Dropping the spannedness assumption in (3.2) we get a similar statement with the inequality

$$
g(S, \operatorname{det} E) \geq 1+c_{2}(E)-r \min \left\{1, \chi\left(\mathscr{O}_{S}\right)\right\},
$$

instead of (3.2.1). The proof is the same; simply replace the inequality $g\left(S, L_{i}\right)$ $\geq q(S)$ with $g\left(S, L_{i}\right) \geq 1-\min \left\{1, \chi\left(\mathscr{O}_{S}\right)\right\}$ [LP, (1.1)].

(ii) Note that for $E$ as in (3.2), $c_{2}(E) \geq \frac{r(r-1)}{2} \geq 1$ and equality implies again $(S, E) \simeq\left(\mathbf{P}^{2}, \mathscr{O}_{\mathbf{P}^{2}}(1)^{\oplus 2}\right)$. Apart from this pair, this inequality is quite a bit stronger than those in (0.1). However it does not hold for indecomposable vector bundles (even very ample) as the pair $\left(\mathbf{P}^{2}\right.$, tangent bundle) shows; indeed, $g(S, \operatorname{det} E)=g\left(\mathbf{P}^{2}, \mathscr{O}_{\mathbf{P}^{2}}(3)\right)=1$, while $c_{2}(E)+1-r=2$.

\section{REFERENCES}

[BaL] E. Ballico and A. Lanteri, Ample and spanned rank-2 vector bundles with $c_{2}=2$ on complex surfaces, Arch. Math. 56 (1991), 611-615.

[BLL] A. Biancofiore, A. Lanteri, and E. L. Livorni, Ample and spanned vector bundles of sectional genera three, Math. Ann. 291 (1991), 87-101.

[F1] T. Fujita, Ample vector bundles of small $c_{1}$-sectional genera, J. Math. Kyoto Univ. 29 (1989), $1-16$.

[F2] _ - On adjoint bundles of ample vector bundles, Complex Algebraic Varieties, Proc., Bayreuth 1990, Lecture Notes in Math., vol. 1507, Springer, Berlin, 1992, pp. 105-112.

[Ha1] R. Hartshorne, Ample vector bundles, Inst. Hautes Études Sci. Publ. Math. 29 (1966), 63-91.

[Ha2] _ Algebraic geometry, Springer-Verlag, Berlin, 1977.

[LM] A. Lanteri and H. Maeda, Adjoint bundles of ample and spanned vector bundles on algebraic surfaces, J. Reine Angew. Math. 433 (1992), 181-199.

[LP] A. Lanteri and M. Palleschi, About the adjunction process for polarized algebraic surfaces, J. Reine Angew. Math. 352 (1984), 15-23. 
[N] A. Noma, Classification of rank-2 ample and spanned vector bundles on surfaces whose zero loci consist of general points, Trans. Amer. Math. Soc. (to appear).

[OSS] Ch. Okonek, M. Schneider, and H. Spindler, Vector bundles on complex projective spaces, Progr. in Math., vol. 3, Birkhäuser, Basel-Boston, 1980.

[R] F. Russo, Some inequalities for ample and spanned vector bundles on algebraic surfaces, preprint.

[So] A. J. Sommese, On manifolds that cannot be ample divisors, Math. Ann. 221 (1976), 55-72.

Dipartimento di Matematica "F. Enriques", Università Via C. Saldini, 50, I-20133 MiLANO, ITALIA

Current address, F. Russo: Institute of Mathematics of the Romanian Academy, P.O. Box 1-764, RO-70700 Bucharest, Romania

E-mail address, A. Lanteri: LANTERI@VMIMAT.MAT.UNIMI.IT 